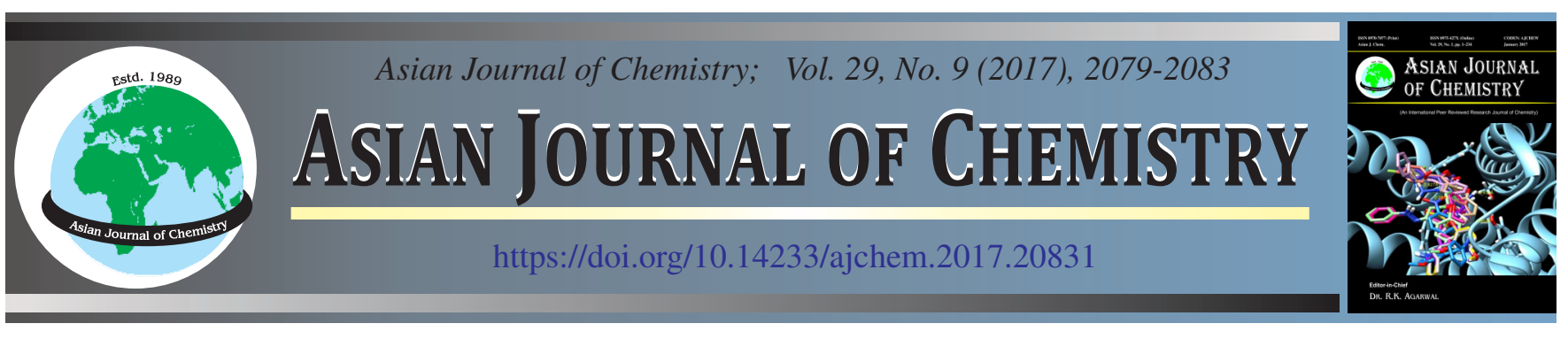

\title{
TLC and HPLC Enantioseparation of Amino Containing Pharmaceutical Using Levofloxacin as Chiral Derivatizing Reagent in Indirect Method
}

\author{
MANOHAR LAL ${ }^{*}$ and JASPREET KAUR
}

Department of Chemistry, Lovely Professional University, Lovely Professional University, Phagwara-144 411, India

*Corresponding author: E-mail: manohar.chem@gmail.com

Received: 19 April 2017;

Accepted: 2 June 2017;

Published online: 15 July 2017;

AJC-18495

Indirect reversed-phase high-performance liquid chromatographic (RP-HPLC) and normal phase thin layer chromatographic method was developed for the separation of enantiomers of (RS)-atenolol using levofloxacin as chiral derivatizing reagent. The indirect separations of the derivatized analytes were performed on $\mathrm{C}_{18}$ column in HPLC and on normal phase plane TLC plates. The effects of organic modifier, mobile phase composition, $\mathrm{pH}$ and temperature on the separation were investigated.

Keywords: Atenolol, Levofloxacine, Chiral derivatizing reagent, RP-HPLC.

\section{INTRODUCTION}

The molecular chirality was first recognized by Louis Pasteur when crystals of sodium ammonium tartrate were mechanically separated by crystallization method [1]. The biological activities of enantiomers can differ surprisingly since the physiological environment of a living being is chiral. The natural and synthetic chiral therapeutic agents exhibit differences in pharmacodynamics and pharmacokinetics properties as they are composed of two enantiomers. Enantiomers also called as optical antipodes or enantiomorphs are non-superimposable mirror-image of each other. The enantiomer responsible for desired therapeutic effect in a body is called eutomer, whereas the other enantiomer which shows unwanted effects such as side effects or toxic effects is termed as distomer $[2,3]$. During new drug development process or asymmetric synthesis of chiral drugs, the chemical and biological entity submissions to the FDA and other regulatory authorities is essentially implemented. To analyze these differences in biological activities of two enantiomers, the development of the chiral analytical method is vitally necessary. Over the last ten years enantiomer separations have become an increasingly important area in the field of pharmaceutical analysis $[4,5]$.

$\beta$-Blockers are 2-hydroxy propyl amines whose enantiomers show a significant difference in their pharmacological effects and activities [6,7]. Atenolol is a very well-known cardio selective $\beta$-blocking agent, which available in the market as a racemic mixture and is used to treat hypertension, coronary heart disease, arrhythmias, sinus tachycardia and myocardial infarction, where it acts preferentially upon the $\beta$-adrenergic receptors in the heart [8]. It causes fewer side effects like cold extremities, dizziness and tiredness, a decrease in heart rate, depression and night mares. Literature reveals that pharmacological action of atenolol is largely confined to (S)-isomer [3] whereas (R)-isomer is inactive in nature. Several reports have been observed in which atenolol has been separated by two different analytical method viz., direct and indirect method of separation. Direct chiral separation of amino acid enantiomers has been done using direct method of separation on TLC in which levofloxacin was used as chiral impregnating agent [6]. Levofloxacin, chemically (S)-9-fluoro-2,3-dihydro-3-methyl10-(4-methyl-1-piperazinyl)-7-oxo-7H-pyrido[1,2,3-de]-1,4benzoxazine-6-carboxylic acid (Fig. 1B), is a fluoroquinolone based chiral drug whose $(S)$-enantiomer act as antimicrobial agent which is mainly used for urinary tract infection $[9,10]$. In this study, levofloxacin was used as chiral derivatizing reagent for the diastereomerization of (RS)-atenolol.

Indirect separation of enantiomers has been achieved by using several chiral derivatizing reagents (CDR) because it can provide an excellent detector response for the trace analysis of biological samples viz., plasma, urine, blood etc. Even sometimes indirect method of separation can provided the better result and resolution than direct method. During past decades, several chromatographic techniques such as gas chromatography (GC), thin layer chromatography (TLC) and high performance liquid chromatography (HPLC) have been widely used for the separation of racemic mixture into individual enantiomers. Among these HPLC has extensively used 
technique for enantiomeric separation. Several advantages such as better reproducibility, flexibility, high resolution, short analysis time, greater sensitivity and detection variation have proved it as successful separation technique in the field of clinical investigations, food analysis, forensic laboratories, biochemistry and pharmaceutical analysis. Moreover, a large number of chiral selectors available enlarge the application of HPLC in the field of chiral separation. However, use of sophisticated instrument, high column cost and more consumption of organic solvents are some disadvantages of HPLC. On the other hand, TLC is cost-effective, simple and inexpensive. Moreover, simultaneous run of standards and samples, easy availability and static off-line detection makes it a viable technique of separation. Therefore, in the search of efficient and effective methods for chiral separation it has become very important to verify the optical purity of chiral drugs and guarantee the therapeutic efficacy and safety of drugs.

Literature reports reveals that the chiral derivatizing reagent (Sanger's reagent) has been used for enantioresolution of six $\beta$-blockers using dinitrophenyl-L-Pro-N-hydroxysuccinimide ester, N-succinimidyl-(S)-2-(6-methoxynaphth-2-yl) propionate by reversed-phase HPLC [4]. Marfey's reagent has been used for derivatization in rat plasma for determination of enantiomers of atenolol by reversed-phase liquid chromatography [5]. Thin layer chromatographic method has been used for enantioseparation of atenolol, propranolol and salbutamol by different approaches of impregnation using $\mathrm{Cu}$ (II)-L-amino acid complexes in ligand exchange chromatography [6]. Chiral derivatization and high-performance liquid chromatography (HPLC) has been used for the preparative resolution of the $\beta$-blocker drug atenolol [7]. Liquid chromatography and other related techniques have been used for separation of some unusual amino acid by using chiral derivatizating reagent [8]. A method has been developed for the simultaneous chiral separation followed by purity determination of chiral switches of atenolol and amLodipine using HPLC [11]. Highly enantioselective Candida antarctica Lipase B (CALB) enzyme catalyzed kinetic resolution of $\beta$-blocker atenolol has been achieved [12]. $\mathrm{C}_{8}$ column has been used for the enantioseparation of atenolol using ligand exchange chromatography [13]. A validated RPHPLC-UV method was developed for simultaneous determination of buparvaquone, atenolol, propranolol, quinidine and verapamil in situ intestinal permeability studies in rat [14]. Direct TLC resolution of the enantiomers of three $\beta$-blockers has been achieved by ligand exchange chromatography with $\mathrm{Cu}$ (II)-L-amino acid complex, using four different approaches [15]. RP-LC resolution of (RS)-atenolol has been performed via diasteriomerization with Marfey's Reagent and its structural variants under conventional and microwave heating [16]. TLC has good scope for the separation of enantiomers of chiral analytes using direct and indirect method by different approaches. These approaches are very simple and cost-effective. The direct method was single step enantioseparation of racemic analytes whereas indirect method involve the formation of diastereomers using optically pure chiral derivatizing reagent (CDR) followed by separation of diastereomers using TLC and HPLC. Reversed phase (RP)-separation on $\mathrm{C}_{18}$ column has been found successful for the separation of diastereomers of amino containing compounds. In present study, we have used TLC and HPLC for the indirect enantioseparation of (RS)atenolol using levofloxacin as chiral derivatizing reagent (CDR). To our best of knowledge, until now, among the $\beta$-blocking chiral drugs, atenolol was first enantio-separated by above said methods.

\section{EXPERIMENTAL}

The drug atenolol (50 mg of each uncoated tablet) and levofloxacin (500 mg each coated tablet) (Fig. 1) was obtained from local market (medical shop) and were manufactured by IPCA laboratories Ltd. (Mumbai, India) and CIPLA Ltd. (Mumbai, India), respectively. Organic solvents like methanol, dichloro methane, acetone, butanol and acetonitrile along with all other reagents like sodium hydrogen phosphate, trifluoro acetic acids, acetic acid and hydrochloric acid were of analytical grade and obtained from Lobachemie (India). The silica gel-G (for TLC) was obtained from Molychem (India). Most of the solution and solvent were prepared in methanol. Double distilled water was used for the preparation of all stock solutions.

All HPLC analyses were performed on a system consisting of a $10 \mathrm{~mL}$ pump head 1000 , a quaternary solvent delivery system, manager 5000 degasser, photodiode array (PDA) detection system 2600, Knauer manual injection valve and Eurochrom operating software and Eurospher $\mathrm{C}_{18}$ column (250 mm, 4.6 mm ID, $5 \mu \mathrm{m}$ ) were from Knauer (Berlin, Germany). All analyses were performed using purified double distilled water. Other equipments used for the analyses were UV-visible absorption spectrophotometer (Shimadzu, Tokyo, Japan), FT-IR spectrometer (Nicolet Shimadzu, Tokyo, Japan), polarimeter, Incubator and digital pH meter. TLC glass slides, developing chambers and other glasswares were used for TLC analyses.
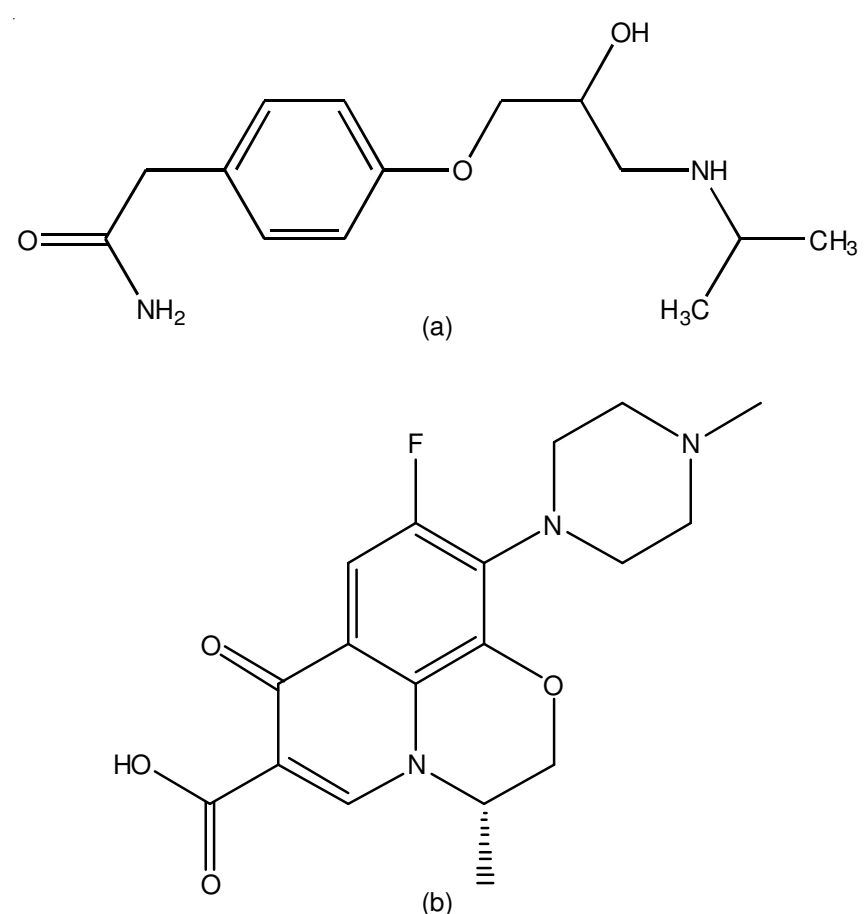

(b)

Fig. 1. Structure of (a) atenolol (analyte) and (b) levofloxacin (chiral selector) 
Extraction and purification of active pharmaceutical ingredient (API) from pharmaceutical formulation: The extraction and purification of atenolol and levofloxacin was done from their tablets as per procedure described earlier. The procedure is discussed as follows:

Extraction of levofloxacin: First of all coatings of 10 tablets of pure levofloxacin (500 mg each) were removed and pulverized using mortar-pestle. Fine powder of the drug was mixed with methanol $(50 \mathrm{~mL})$ to extract the API. Afterward solution was filtered followed by evaporation of solvent on rota-vapor and left the residue in round bottom flask to cool until crystals appeared. Then recrystallization of the extracted compound was carried out in methanol-chloroform mixture (7:3) and recorded the yield. The purity of the extracted drug was corroborated by UV-visible spectrum, infrared spectrum and melting point determination.

Extraction of atenolol: The extraction and purity of 14 uncoated tablets of pure atenolol (50 mg each) was achieved using above mentioned procedure.

Preparation of stock solutions: All stock solutions of racemic $\beta$-blocker i.e., (RS)-atenolol $\left(10^{-2} \mathrm{M}\right)$ and $(\mathrm{S})$ levofloxacin $\left(10^{-2} \mathrm{M}\right)$ were prepared in methanol and $0.1 \mathrm{M}$ $\mathrm{NaHCO}_{3}$ for derivatization reaction. Solution of $\mathrm{NaHCO}_{3}(0.1$ $\mathrm{M})$ was prepared in purified water.

Preparation of plane TLC plates: An unmodified TLC plates were prepared by spreading slurry of silica gel-G (10 g) dissolved in double distilled water $(20 \mathrm{~mL})$. The TLC plates were activated in hot air oven for $4 \mathrm{~h}$ at $105^{\circ} \mathrm{C}$.

\section{Mobile phases for chromatographic separation}

For TLC: Following mobile phases with different ratio were tested for the development of TLC plates: acetonitrile$\mathrm{MeOH}-\mathrm{H}_{2} \mathrm{O}(6: 1: 1)$, acetonitrile- $\mathrm{MeOH}-\mathrm{H}_{2} \mathrm{O}-\mathrm{CH}_{2} \mathrm{Cl}_{2}$ (7:1:1:1), $n$-butanol-acetic acid-water (4:1:1), $\mathrm{MeOH}-\mathrm{CH}_{2} \mathrm{Cl}_{2}$ (4:6), $\mathrm{CH}_{3} \mathrm{CN}-\mathrm{CH}_{3} \mathrm{OH}-\mathrm{CH}_{2} \mathrm{Cl}_{2}(3: 3: 4), \mathrm{C}_{2} \mathrm{H}_{5} \mathrm{OH}-\mathrm{CHCl}_{3}(2: 1)$.

For HPLC: Following solvent mixtures were used as mobile phase: Mobile phase (I) eluent (A) [acetonitrile (100
$\mathrm{mL})$, water $(900 \mathrm{~mL})$ and tetrahydrofuran $(\mathrm{THF})(1 \mathrm{~mL})]$ and eluent (B) [acetonitrile $(800 \mathrm{~mL})$, water $(200 \mathrm{~mL})$ and trifluoroacetic acid (1 mL)]; Mobile phase (II) eluent (C), [methanol $(100 \mathrm{~mL})$, water $(900 \mathrm{~mL})$ and trifluoroacetic acid $(1 \mathrm{~mL})]$ and eluent (D) [methanol $(800 \mathrm{~mL})$, water $(200 \mathrm{~mL})$ and trifluoroacetic acid $(1 \mathrm{~mL})]$.

Different gradients of mobile phases i.e. $25 \% \mathrm{~A}-100 \%$ B, $50 \%$ A-100\% B, $75 \%$ A-100\% B and $100 \%$ A-100\% B; $45 \mathrm{~min}$ each) $60 \mathrm{~min}$ each were tested for separation of diastereomeric pairs. A flow rate of $1 \mathrm{~mL} / \mathrm{min}$ was used throughout the study. The wavelength $266 \mathrm{~nm}$ was used for the detection of eluted peaks.

Synthesis of diastereomers of atenolol: (RS)-Atenolol was derivatizated with chiral reagent levofloxacin using dicyclohexylcarbodiimide (DCC) as dehydrating agent. The derivatization procedure is discussed as follows:

The solution of (RS)-atenolol $\left(10^{-2} \mathrm{M}\right)$ was added to solution of levofloxacin $\left(10^{-2} \mathrm{M}\right)$ in dichloromethane (DCM). The dicyclohexylcarbodiimide (DCC) $(2 \mathrm{mM})$ was added to this solution. The reaction mixture was kept on magnetic stirrer at $40-50{ }^{\circ} \mathrm{C}$ for $2.5 \mathrm{~h}$ with constant stirring. The completion of derivatization reaction (i.e. reaction progress) was carried out by continuous TLC monitoring before workup. After completion of reaction the mixture was cooled at room temperature followed by filtration and evaporation on rotavapor. A synthetic scheme of diastereomeric formation was shown in Fig. 2.

\section{RESULTS AND DISCUSSION}

In proposed method a simple, economical, accurate and rapid approach was used for the enantiomeric separation of racemic atenolol using levofloxacin as chiral derivatizing reagent (CDR). The diastereomers of racemic atenolol were prepared on treating it with levofloxacin in presence of dicyclohexylcarbodiimide (dehydrating agent). The diastereomers so prepared were separated using liquid chromatographic techniques i.e. TLC and HPLC.

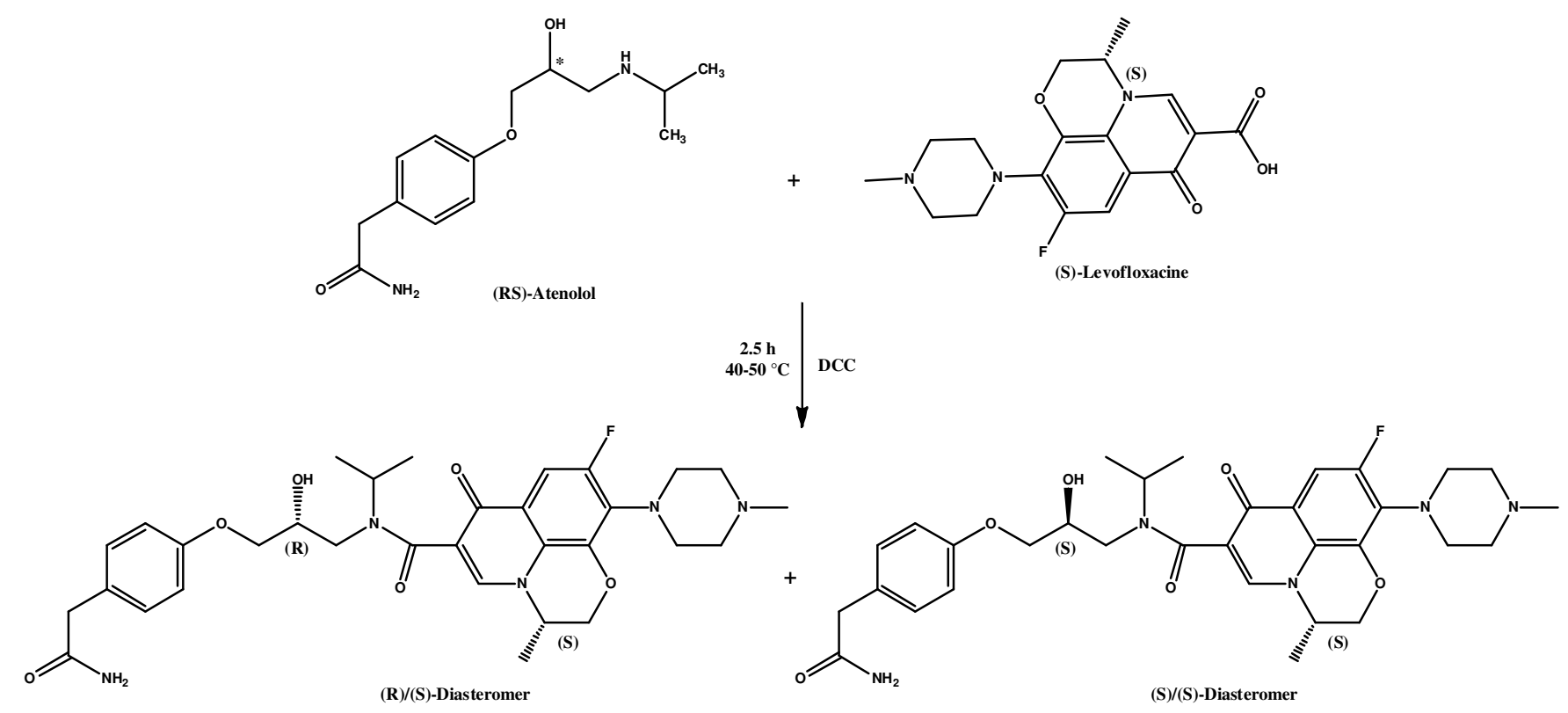

Fig. 2. Scheme for the synthesis of diastereomers of (RS)-atenolol with chiral selector levofloxacin 


\section{HPLC and TLC analytical method development}

Selection of HPLC column: Reversed phase $\mathrm{C}_{18}$ column is used widespread in chromatographic separation due to their reproducibility of results and wide applications. Several amino containing pharmaceuticals have already been separated on $\mathrm{C}_{18}$ column. Hence a $\mathrm{C}_{18}$ column was initially considered for the separation of $\beta$-blocker enantiomers using indirect method.

Optimization of mobile phase: In the present study, various combinations of mobile phase were executed in under reversed phase systems.

Following mobile phase combinations were used: (I) eluent (A) [acetonitrile $(100 \mathrm{~mL})$, water $(900 \mathrm{~mL})$ and tetrahydrofuran (THF) $(1 \mathrm{~mL})$ ] and eluent $(B)$ [acetonitrile $(800 \mathrm{~mL})$, water $(200 \mathrm{~mL})$ and trifluoroacetic acid (1 mL)]; (II) eluent (C), [methanol $(100 \mathrm{~mL})$, water $(900 \mathrm{~mL})$ and trifluoroacetic acid $(1 \mathrm{~mL})]$ and eluent (D) [methanol $(800 \mathrm{~mL})$, water $(200 \mathrm{~mL})$ and trifluoroacetic acid $(1 \mathrm{~mL})]$. The gradient mode of elution was used throughout the study. Different gradients of mobile phases were tried and a linear gradient of $100 \%$ A to $100 \%$ B (in mobile phase I) and $100 \% \mathrm{C}-100 \% \mathrm{D}$ (in mobile phase II) were found to be successful. Acetonitrile was observed as better elution system when compared to methanol as broader peaks and larger retention time was observed in later organic modifier. All mobile phases were degassed by passing nitrogen and sonication before use. All mobile phases were filtered through a $0.45 \mu \mathrm{m}$ filter prior to HPLC run. The injection volume of each sample was $20 \mu \mathrm{L}$. Detection was made through a PDA detector at $266 \mathrm{~nm}$. Experiments were carried out at a fixed temperature of $25{ }^{\circ} \mathrm{C}$. The flow rate was $1.0 \mathrm{~mL} / \mathrm{min}$ throughout the study. The total run time was limited to $60 \mathrm{~min}$ in each run.

Synthesis of diastereomers: Active pharmaceutical ingredient (API) of atenonol was extracted from its pharmaceutical formulation. Methanol was used to extract the pulverized drug and purity was ascertained by UV and IR analysis. Extracted API so obtained is further used for the derivatization. LEVO was used as CDR to diastereomerize the analyte (RS)-Atl in presence of dehydrating agent dicyclohexylcarbodiimide. The completion of the derivatization was confirmed by sequential TLC checking. The appearance of two distinct spots in reaction mixture line/spot indicates completion of reaction and formation of two diastereomers.

Enantioseparation on thin layer chromatography (TLC): Enantioseparation along with actual chromatograms and their respective results are shown below:

(1) A solution of $\left(10^{-2} \mathrm{M}\right)$ reaction mixture $(\mathrm{R} / \mathrm{S}, \mathrm{S} / \mathrm{S})$ was prepared in $\mathrm{MeOH}$. Then a spot was marked on plain TLC plate and mixture of solvents like $\mathrm{MeCN}-\mathrm{MeOH}-\mathrm{H}_{2} \mathrm{O}(6: 1: 1)$ was used as developing solvent. Afterward, the TLC plate was dried at room temperature and placed it in an iodine chamber for spot location.
Two different spots are located at different $R_{\mathrm{f}}$ value; $\mathrm{R}_{\mathrm{f}}$ for $1^{\text {st }}$ spot $=3.1 / 5.5=0.56 ; R_{f}$ for $2^{\text {nd }}$ spot $=4.7 / 5.5=0.854$.

(2) The solution of extracted chiral selector (LEVO) $\left(10^{-2}\right.$ $\mathrm{M})$ was prepared in $\mathrm{MeOH}$. A spot was marked on plain TLC plate and mixture of solvents like $\mathrm{MeCN}-\mathrm{MeOH}-\mathrm{H}_{2} \mathrm{O}$ (6:1:1) was used as developing solvent. Afterward, the TLC plate was dried at room temperature and placed it in an iodine chamber for spot location.

One spot are located at different $R_{f}$ value. $R_{f}$ for $1^{\text {st }}$ spot Levofloxacin $=3.7 / 5.8=0.63$. This confirms absence of any type of impurities in extracted chiral selector.

(3) The solutions of (RS)-atenolol and reaction mixture (containing R/S, S/S-diastereomers) $\left(10^{-2} \mathrm{M}\right.$ each) were prepared in $\mathrm{MeOH}$. This solution was spotted on plain TLC plate and spotted TLC was dipped for development in a mixture of $n$-butanol-acetic acid-water with the ratio 4:1:1. Afterward, the TLC plate was dried at room temperature and placed it in an iodine chamber for spot location.

In right hand side (RHS): reaction mixture was spotted and obtained two different spot on TLC at different $\mathrm{R}_{\mathrm{f}}$ value and in left hand side (LHS) one spot was obsevered. For reaction mixture $(\mathrm{RHS}) \mathrm{R}_{\mathrm{f}}$ value $1^{\text {st }}$ spot $=2.7 / 5=0.54, \mathrm{R}_{\mathrm{f}}$ value for $2^{\text {nd }}$ spot $=4.2 / 5=0.84$; For atenolol (LHS) $R_{f}$ value $=2.9 / 5=0.58$.

Enantioseparation on HPLC: The retention times $\left(t_{R}\right)$ retention factor $(k)$, separation factor $(\alpha)$ and resolution (Rs) of the resolved diastereomers using successful mobile phase I are summarized in Table-1. A full chromatogram of the run showing the enantioseparation of (RS)-Atl (two similar peaks) and one extra peak indicate the peak of excess CDR is shown in Fig. 3. Enantioseparation of (RS)-atenolol was confirmed by comparing the peak area ratios of two similar peaks.

Chromatographic parameters concluded in Table-1 like separation factor is greater than 1 and resolution greater than 1.5 indicates this separation is a baseline separation of enantiomer. However, retention factor values are large which is undesirable and somehow time consuming. These values should be as small as possible to save the analysis time.

Effect of temperature and pH: Earlier studies on enantioseparation of different chiral drugs by indirect method of separation using chiral derivatizating reagent on TLC plate showed to affect the enantioseparation on variation in temperature and $\mathrm{pH}$. The chromatograms on TLC were developed at $15 \pm 2,20 \pm 2$ and $25 \pm 2{ }^{\circ} \mathrm{C}$ to study the effect of temperature on separation. Separation was found to be temperature dependent as the temperature lowers at $15 \pm 2{ }^{\circ} \mathrm{C}$ then the separation was good and spots were clearly visible. But as the temperature increases above $25 \pm 2{ }^{\circ} \mathrm{C}$ then spots were elongated like eight shaped spots. It may be due to fast movement of analyte on chiral stationary phase (chiral TLC) resulting in lesser inter-

TABLE-1

CHROMATOGRAPHIC PARAMETERS FOR THE SEPARATION OF DIASTEREOMERS OF (RS)-ATENOLOL PREPARED WITH LEVOFLOXACIN

\begin{tabular}{cccccc}
\hline Peaks & $\begin{array}{c}\text { Retention } \\
\text { time }\left(\mathrm{t}_{\mathrm{R}}\right)\end{array}$ & Peak area & Width & $\begin{array}{c}\text { Retention factor } \\
(\mathrm{K} 1 \text { and K2) }\end{array}$ & $\begin{array}{c}\text { Separation factor } \\
(\alpha=\mathrm{K} 2 / \mathrm{K} 1)\end{array}$ \\
\hline $\mathrm{t} 1$ & 42.0 & 95.85 & 0.449 & 19.30 & 21.37 \\
$\mathrm{t} 2$ & 46.1 & 96.06 & 0.454 & 8.2 & 21.107 \\
\hline
\end{tabular}




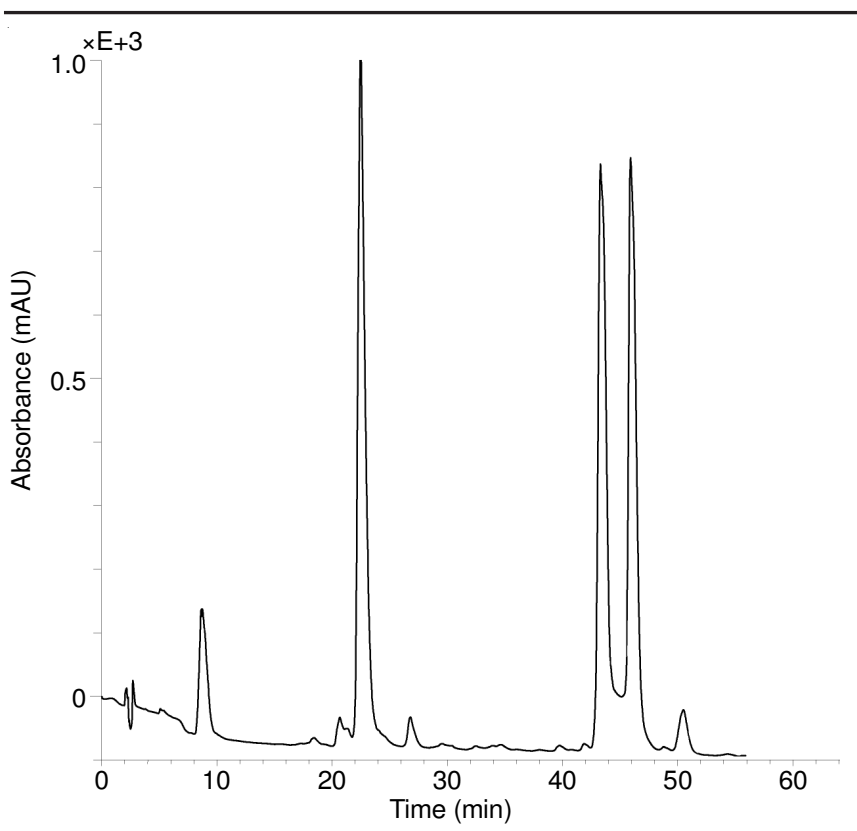

Fig. 3. Chromatogram showing resolution of diastereomers of (RS)-atenolol prepared with levofloxacin

action time with chiral selector and other factor may be solvent system having two or more than two components, if one of the component is volatile in nature then chances of evaporation will be more at higher temperature which in turn provide different mobile phase composition rather than original. Thus, volatile nature of the component affects the chiral interaction of analyte and chiral selector. In case of HPLC, enantioseparation was performed at $25^{\circ} \mathrm{C}$ due to non-availability of column oven in HPLC.

In literature, $\mathrm{pH}$ of mobile phase and reaction conditions were also found to influence the enantioseparation. Solutions of the chiral selectors were adjusted to $\mathrm{pH}$ range 6-9 to find out the optimum condition for good resolution. Most of the successful separations were achieved in basic $\mathrm{pH}(\mathrm{pH}$ around 8).

\section{Conclusion}

The present work describes improved analytical method for the synthesis of diastereomers by the reaction of atenolol and levofloxacine using N,N'-dicyclohexylcarbodiimide (DCC) which is used as dehydrating agent for the preparation of amide. The amidic diastereomers so formed were separated on TLC. The simplicity, flexibility, versatility and sensitivity make the TLC technique more advantageous for the enantio- separation of diasteriomer via indirect method. There is a clear evidence of TLC being a complimentary technique to HPLC as it is inexpensive, simple, less time consuming and easily approachable, but for diastereomeric separation or indirect separation HPLC provide excellent separation by gradient mode of separation and detection can be achieved using diverse class of detector. The developed method can be applied to recover the pure enantiomer for further use in pharmaceuticals and asymmetric synthesis.

\section{ACKNOWLEDGEMENTS}

The authors are thankful to Lovely Professional University for providing the financial and instrumental support.

\section{REFERENCES}

1. A.G. Gilman, L.S. Goodman and A. Gilman, Goodman and Gilman's The Pharmacological Basis of Therapeutics, Macmillan, New York (1980).

2. T. Walle, J.G. Webb, E.E. Bagwell, U.K. Walle, H.B. Daniell and T.E. Gaffney, Biochem. Pharmacol., 37, 115 (1988); https://doi.org/10.1016/0006-2952(88)90763-0.

3. W.T. Comer and A.W. Gomoll, ed.: A. Burger, Medicinal Chemistry, Part II, Wiley-Interscience, New York, Cahp. 39, p. 1019 (1970).

4. R. Bhushan and S. Tanwar, Biomed. Chromatogr., 23, 1291 (2009); https://doi.org/10.1002/bmc. 1252.

5. R. Bhushan and S. Tanwar, Biomed. Chromatogr., 23, 787 (2009); https://doi.org/10.1002/bmc.1187.

6. R. Bhushan and S. Tanwar, J. Chromatogr. A, 1217, 1395 (2010); https://doi.org/10.1016/j.chroma.2009.12.071.

7. M.J. Wilson, K.D. Ballard and T. Walle, J. Chromatogr. A, 431, 222 (1988); https://doi.org/10.1016/S0378-4347(00)83089-3.

8. I. Ilisz, A. Aranyi and A. Péter, J. Chromatogr. A, 1296, 119 (2013); https://doi.org/10.1016/j.chroma.2013.03.034.

9. S.C. Sweetman, Martindale, The Complete Drug Reference, Pharmaceutical Press, edn 33 (2002).

10. G. Tamilarasi, T. Vetrichelvan, D. Vekappayya, R. Tharabai and K. Yuvarajan, J. Pharm. Pharm. Sci., 6, 646 (2014).

11. V. Kannappan and S.S. Mannemala, J. Pharm. Biomed. Anal., 120, 221 (2016); https://doi.org/10.1016/j.jpba.2015.12.048.

12. I.T. Lund, P.L. Bøckmann and E.E. Jacobsen, Tetrahedron, 72, 7288 (2016); https://doi.org/10.1016/j.tet.2016.02.018.

13. T. Alizadeh, Sep. Purif. Technol., 118, 879 (2013); https://doi.org/10.1016/j.seppur.2013.08.032.

14. G. Venkatesh, S. Ramanathan, S.M. Mansor, N.K. Nair, M.A. Sattar, S.L. Croft and V. Navaratnam, J. Pharm. Biomed. Anal., 43, 1546 (2007); https://doi.org/10.1016/j.jpba.2006.11.013.

15. R. Bhushan and S. Tanwar, Chromatographia, 70, 1001 (2009); https://doi.org/10.1365/s10337-009-1216-7.

16. R. Bhushan and S. Tanwar, Chromatographia, 68, 849 (2008); https://doi.org/10.1365/s10337-008-0758-4. 